\title{
Grand Challenges in Oncology
}

\section{Giuseppe Giaccone*}

Medical Oncology Branch, National Cancer Institute, Bethesda, MD, USA

${ }^{*}$ Correspondence: giacconeg@mail.nih.gov

Since the declaration of war against cancer in the National Cancer Act of 1971 by then U.S. President Richard Nixon, great progress has been made in several areas, but it is clear that cancer is a more complex disease than what we had anticipated. Despite the high cure rates achieved in some hematological malignancies and pediatric tumors, progress in the most common adult solid tumors has been slower and less impressive. However, there are important improvements in the understanding of the causation of cancer and the molecular mechanisms by which cancer develops and disseminates, which have allowed a better understanding of the biology and the definition of new treatment strategies. The understanding of these basic mechanisms has allowed the development of a number of more targeted anticancer agents that are exquisitely active in patients whose tumors have specific genetic abnormalities.

\section{PREVENTION AND EARLY DIAGNOSIS}

Overall, an estimated 12.7 million new cancer cases and 7.6 million cancer deaths occurred in 2008 , with $56 \%$ of new cancer cases and $63 \%$ of the cancer deaths occurring in the less developed regions of the world, as assessed by the GLOBOCANC, which investigated the worldwide incidence and mortality from 27 cancers in 182 countries in 2008 (Ferlay et al., 2010). The most commonly diagnosed cancers worldwide are lung (1.61 million, $12.7 \%$ of the total), breast (1.38 million, $10.9 \%$ ), and colorectal cancers (1.23 million, 9.7\%). The most common causes of cancer death are lung cancer (1.38 million, $18.2 \%$ of the total), stomach cancer $(738,000$ deaths, $9.7 \%)$, and liver cancer $(696,000$ deaths, $9.2 \%)$. Cancer is neither rare anywhere in the world, nor mainly confined to highresource countries. Striking differences in the patterns of cancer from region to region are observed. Progress in all these major cancer killers has come from prevention, screening, and early detection, whereas the treatment of cancers in advance stage has undergone significant improvements but with a much lesser effect on overall survival.

Mammography screening every 2 years is presently advised in women of 50 years of age, up to 75 (US Preventive Services Task Force, 2009), although several strongly recommend to start of age 40 .

Screening for cancer of the cervix has been in use for years and colonoscopy has been more recently advised to prevent colorectal cancer. PSA screening for prostate cancer is still highly controversial, but used by many. Very recently the results of the National Lung Screening trial (NLST) have indicated that low dose helical CT scan is able to reduce mortality in heavy smokers, compared to chest X-ray. The development of imaging techniques or methods to assess with precision the risk of developing tumors is of paramount importance in the reduction of the burden of cancer. Prevention is of course also very important and epidemiological studies have led to the adoption of preventing measures that have had a major impact in the survival and incidence of specific tumor types. As an example the banning of cigaret smoke has determined the reduction of lung cancer in the United States. With the increasing use of low dose spiral CT scans for detection of early lung cancer we will be seeing an increasing number of small nodules and lung lesions that will pose challenges to the diagnosis and treatment that have so far not been faced. The challenge in many tumors is the development of more sensitive tests for early diagnosis, using either imaging or molecular studies. The ability to detect single molecules using very sensitive approaches is probably going to allow the use of specific tests in the future.

\section{TREATMENT OF EARLY DISEASE}

Treatment of solid tumors at a localized stage has remained surgery for the most part, but we have seen changes in several tumor types, to include less mutilating operations, and organ preserving therapies, e.g., in breast cancer, rectal cancer, larynx cancer. For example, mastectomy has been replaced by smaller resections, to which radiation has been added. Chemoradiotherapy has become standard in rectal and anal cancer and in some forms of head and neck cancer, with a significant cure rate, which is comparable to that of surgery. In the years to come, the use of alternative treatment strategies, to limit the extent of surgery, together with lesser invasive surgeries, will likely make a large impact to how locally advanced tumors will be handled.

In several solid tumors adjuvant treatments have become standard, and can improve significantly survival, such as in breast, colon, and lung cancer. A remarkably large number of patients are candidates for these treatments and refinements of adjuvant therapies with increased activity and lesser long-time side effects have become part of the research programs for these and other diseases.

\section{HEREDITARY CANCERS}

Several forms of cancer have been identified where a genetic predisposition plays an important role in the causation of the cancer. BRCA1 and BRCA2 germ line mutations are responsible for $90 \%$ of hereditary forms of breast and ovarian cancers. Lynch syndrome (hereditary non-polyposis colon cancer, HNPCC) is caused by mutations in one of six genes involved in DNA mispatch repair, and is responsible for about $2-3 \%$ of colon cancer cases. Another $1 \%$ of colon cancers is caused by mutations in the APC gene, which is associated with familial adenomatous polyposis. Retinoblastoma is hereditary and due to mutations in the $\mathrm{Rb}$ gene in about $40 \%$ of cases of retinoblastomas. Mutations in several other genes also predispose to a variety of cancers. For example LKB1 mutations associated with Peutz-Jeghers syndrome predispose to gastric cancer as well as other cancer. The Lee-Fraumeni syndrome, caused by $\mathrm{p} 53$ mutations, also predisposes patients to a variety of tumor types. 
More recently low penetrance SNPs have also been implicated in determining a higher risk of cancer, as identified by GWAS studies (Freedman et al., 2011).

Counseling and specific follow up and treatment strategies for patients affected by genetic predisposition to cancer and their families, have been developed and have become an important part of comprehensive cancer care.

\section{VIRUS INDUCED CANCER AND PREVENTION}

Since the introduction of the theories of cancer inducing viruses (cancer virus or oncovirus), hepatitis viruses, EBV, HPV, and many other viruses have been unequivocally identified as initiators of carcinogenesis. Prevention strategies and vaccination strategies have been developed for several of these diseases. The development of an HPV vaccine for cervical cancer has been a major breakthrough that has led to vaccination programs in high risk women, and may have broader implications in other HPV mediated cancers, such as head and neck HPV induced carcinomas. Analogously, prevention of hepatitis induced liver cancer has broad impact in the development of the disease in geographic areas where hepatitis virus infection is endemic, such as in large areas of Asia and Africa.

\section{TREATMENT OF ADVANCED DISEASE}

Despite the success obtained in hematological malignancies, and that obtained in testicular cancer, which can be cured with chemotherapy in the vast majority of cases even at advanced stage, most solid tumors have not witnessed such improvement in cure rates or survival. The introduction of targeted therapies for specifically defined subsets of patients has made a major impact in the treatment of diseases where standard therapies had poor impact, such as CML and GIST with imatinib, EGFR tyrosine kinase inhibitors for EGFR mutant non-small cell lung cancer, ALK inhibitors for non-small cell lung cancers with ALK translocations, BRAF inhibitors for BRAF mutant melanoma, Her2 inhibitors for Her2 amplified breast cancer, to cite a few examples. Other targeted agents have however been successfully developed, which target the microenvironment and angiogenesis rather than the cancer cells, and where patient selection has been challenging.
Important improvements have also recently been seen with the use of immunological approaches. Important examples are provenge for castrate-resistant prostate cancer (Plosker, 2011) and ipilimumab for advanced melanoma (Robert et al., 2011). Vaccines and other immunotherapies are presently being investigated in other solid tumors.

The way forward appears however to be in combinations of different drugs and approaches, in the attempt to utilize different mechanisms of action that tackle one or more cancer pathways, and to overcome the several potential mechanisms of resistance. The challenge is represented by overlapping toxicities of the different drugs. Patient selection for particular combinations remains a challenge and improvement is needed in this regard. Preclinical testing of combinations will need to be perfected, since the current methods for in vitro and in vivo testing of combinations are poorly predictive of additive or synergistic interactions.

\section{ADVANCES IN BASIC SCIENCE}

There has been tremendous progress in the understanding of the cancer process, transformation, progression, and metastasis. This has led to better strategies to approach a number of cancers and potentially novel therapeutics. The theory of the cancer stem cell has paved the way to the development of novel strategies aimed at the eradication of cell clones that are essentially resistant to standard therapies and that potentially have markers that can be targeted specifically (e.g., Wnt, Notch, hedge hog pathways among others; Clevers, 2011). Drug development is in progress in the attempt to target specific cancer stem cell molecules. Clinical trials of cancer stem cell targeted agents may require different patient populations or endpoints as compared to those testing conventional drugs, since only a small population of cells will be targeted by these agents, which might be best suited after cytoreductive treatments such as surgery or induction chemotherapy have been applied.

With the pace of whole genome sequencing, functional genomics, and proteomics applied to the cancer, we are presently facing an enormous amount of data that is being generated (Parsons et al., 2008). In order to make sense of this mass of data, novel bioinformatics tools and strategies to store and analyze the information need to be developed. Technology has drastically reduced the amount of time and money needed to study cancer at genome-wide level, but challenges remain in the development of tools to analyze data in a timely fashion and in storing of large information datasets. The discovery of novel targets for treatment, prognostic factors and potentially genetic predisposition factors are being pursued by large sequencing projects in several tumor types. Large initiatives are bringing to light the discovery of genetic abnormalities in the most common tumor types.

\section{NEW PERSPECTIVES}

Certainly there is a lot of fervor in the definition of cancer genetics and this will probably lead to more discoveries of treatments that may be specifically target vulnerable functions within the cancer cell. However, systemic treatment of advanced disease is unlikely to produce major improvement in survival of the most common cancer types, since these common solid tumors usually have redundant mutations that make the tumor heterogeneous and very plastic and dynamic, with development of drug resistance mechanisms in a very quick fashion. Although important discoveries have been made with targeted therapies, they really substantially affect only a minority of the cancer types (e.g., CML, GIST, BRAF mutant melanoma, EGFR mutant lung cancer, ALK positive lung cancer, etc.). Although it is possible that more activating mutations and targetable genetic defects be discovered, most solid tumors are heterogeneous and therefore escape mutants will likely be present from start, and/or emerge quickly following drug pressure.

Thus, major improvements in overall survival will likely continue to rely heavily upon early detection and prevention strategies.

\section{FRONTIERS IN ONCOLOGY}

This new open access journal has one of the major goals to be the forum for publications in the most important areas of cancer research, spanning from diagnosis, and prevention to treatment of early and advanced cancer and drug development, to the most cutting edge technologies that can be implemented to study cancer and improve its treatment. The fast pace of research and its 
ever increasing scope and breath, require a comprehensive forum that allows both the depth of research and a broad readership. This will be accomplished by a large series of subspecialties within Frontiers in Oncology, which span the major areas of disease-oriented research, epidemiology and prevention, treatment, and basic research.

\section{REFERENCES}

Clevers, H. (2011). The cancer stem cell: premises, promises and challenges. Nat. Med. 17, 313-319.

Ferlay, J., Shin, H. R., Bray, F., Forman, D., Mathers, C., and Parkin, D. M. (2010). Estimates of worldwide burden of cancer in 2008: GLOBOCAN 2008. Int. J. Cancer 127, 2893-2917.

Freedman, M. L., Monteiro, A. N., Gayther, S. A., Coetzee, G. A., Risch, A., Plass, C., Casey, G., De Biasi, M.,
Carlson, C., Duggan, D., James, M., Liu, P., Tichelaar, J. W., Vikis, H. G., You, M., and Mills, I. G. (2011). Principles for the post-GWAS functional characterization of cancer risk loci. Nat. Genet. 43, 513-518.

Parsons, D. W., Jones, S., Zhang, X., Lin, J. C., Leary, R. J., Angenendt, P., Mankoo, P., Carter, H., Siu, I. M., Gallia, G. L., Olivi, A., McLendon, R., Rasheed, B. A., Keir, S., Nikolskaya, T., Nikolsky, Y., Busam, D. A. Tekleab, H., Diaz, L. A. Jr., Hartigan, J., Smith, D. R., Strausberg, R. L., Marie, S. K., Shinjo, S. M., Yan, H., Riggins, G. J., Bigner, D. D., Karchin, R., Papadopoulos, N., Parmigiani, G., Vogelstein, B., Velculescu, V. E., and Kinzler, K. W. (2008). An integrated genomic analysis of human glioblastoma multiforme. Science 321, 1807-1812.

Plosker, G. L. (2011). Sipuleucel-T: in metastatic castration-resistant prostate cancer. Drugs 71, 101-118.

Robert, C., Thomas, L., Bondarenko, I., O'Day, S., Weber, J., Garbe, C., Lebbe, C., Baurain, J. F., Testori, A., Grob, J.J., Davidson, N., Richards, J., Maio, M., Hauschild,A.,
Miller, W. H. Jr., Gascon, P., Lotem, M., Harmankaya, K., Ibrahim, R., Francis, S., Chen, T. T., Humphrey, R., Hoos, A., and Wolchok, J. D. (2011). Ipilimumab plus dacarbazine for previously untreated metastatic melanoma. N. Engl. J. Med. 364, 2517-2526.

US Preventive Services Task Force. (2009). Screening for breast cancer: U.S. Preventive Services Task Force recommendation statement. Ann. Intern. Med. 151, 716-726, W-236.

Received: 11 July 2011; accepted: 22 August 2011; published online: 08 September 2011.

Citation: Giaccone G (2011) Grand Challenges in Oncology. Front. Oncol. 1:26. doi: 10.3389/fonc.2011.00026

Copyright $\odot 2011$ Giaccone. This is an open-access article subject to a non-exclusive license between the authors and Frontiers Media SA, which permits use, distribution and reproduction in other forums, provided the original authors and source are credited and other Frontiers conditions are complied with. 